\title{
Research on Parametric Design of Hydraulic Retarder Based on Multi- Field Coupling of Heat, Fluid and Solid
}

\author{
Kuiyang Wang*, Jinhua Tang and Guoqing Li
}

School of Automotive and Traffic Engineering, Jiangsu University of Technology, Changzhou, 213001, China

\begin{abstract}
In order to optimize the design method and improve the performance of hydraulic retarder, the numerical simulation of multi-field coupling of heat, fluid and solid is carried out to hydraulic retarder, based on the numerical computation and algorithm of heat-fluid coupling and fluid-solid coupling. The computation models of heat-fluid coupling and fluid-solid coupling of hydraulic retarder are created. The three dimensional model of hydraulic retarder is established based on CATIA software, and the whole flow passage model of hydraulic retarder is extracted on the basis of the three dimensional model established. Based on the CFD calculation and the finite element numerical simulation, the temperature field, stress field, deformation and stress state are analysised to hydraulic retarder in the state of whole filling when the rotate speed is $1600 \mathrm{r} / \mathrm{min}$. In consideration of rotating centrifugal force, thermal stress and air exciting vibration force of blade surface, by using the sequential coupling method, the flow field characteristics of hydraulic retarder and dynamic characteristics of blade structure are analysised and researched based on multi-field coupling of heat, fluid and solid. These provide the theoretical foundation and references for parametric design of hydraulic retarder.
\end{abstract}

Keywords: Hydraulic retarder, heat-fluid coupling, fluid-solid coupling, numerical simulation, parametric design.

\section{INTRODUCTION}

Hydraulic retarder is one of the auxiliary braking devices for modern heavy vehicles, which extend the service life of the brake and tyre by diverting brake load and reducing the number of braking. Through the use of hydraulic retarder, the comfort performance, reliability and security of vehicle can be improved [1]. When the hydraulic retarder works, the oil within hydraulic retarder will move along the axial direction and the blade direction of driving wheel under the driving of driving wheel. The oil which moves along blade direction is thrown to the fixed wheel, the blades of fixed wheel return function on the oil and act on the driving wheel through the oil. These block the rotation of driving wheel and the braking torque is produced. Thus, the speed of driving wheel is reduced to realize effect of retarder braking [2].

In this process, the loading conditions of blade are very complex. It is mainly affected by some factors such as changing centrifugal force, fluid exciting force, liquid thermal deformation, etc. This will lead to the forced vibration of blade, and the vibration for a long time easily leads to fatigue failure of blade. At present, the study on strength of hydraulic retarder's blade is mainly concentrated in considering the effect of centrifugal force, without considering the effects of temperature and fluid on the deformation of blade. In fact, the rising of temperature of oil and the variation of fluid pressure will cause thermal deformation of the blades. In this paper, by using the sequential multi-field coupling method based on heat-fluid coupling and fluid-solid coupling, the temperature field, pressure field, stress and strain of hydraulic retarder are analysised. These provide the theoretical foundation and references for parametric design of hydraulic retarder.

\section{ESTABLISHMENT OF MATHEMATICAL MODEL OF COUPLING}

Coupling is a kind of phenomenon on the interaction and mutual influence between two or more than two physical quantities [3]. The coupling of hydraulic retarder is mainly reflected in the mutual influence and mutual superposition of flow field, temperature field and structure field, and the multi-field coupling is formed eventually [4].

\subsection{Mathematical Model of Heat-Fluid Coupling}

The essence of heat-fluid coupling for hydraulic retarder is the interaction and mutual influence between temperature field and flow field [5]. The temperature field changes the velocity of fluid through influencing the characteristics of fluid such as the density and viscosity of oil. At the same time, the distribution of flow field decides the distribution trend of temperature field [6]. In the study of convective heat transfer, the fluid is usually regarded as continuous fluid. Taking a micro volume of fluid (i.e., control volume) as the object of study, considering the dynamics and thermodynamics properties of fluid in the control volume, the basic differential equations which are generally applicable are obtained. The basic control equations required on solving flow field and temperature field of hydraulic retarder are shown as follows [7].

The continuity equation: 


$$
\frac{\partial(\rho \mu)}{\partial x}+\frac{\partial(\rho v)}{\partial y}+\frac{\partial(\rho \omega)}{\partial z}=0
$$

where $\rho$ is the density of fluid.

The momentum equation:

$$
\begin{aligned}
& \frac{\partial\left(\rho \mu^{2}\right)}{\partial x}+\frac{\partial(\rho \mu \nu)}{\partial y}+\frac{\partial(\rho \mu \omega)}{\partial z}=-\frac{\partial p}{\partial x}+\frac{\partial \sigma_{x x}}{\partial x}+\frac{\partial \sigma_{x y}}{\partial y}+\frac{\partial \sigma_{x z}}{\partial z} \\
& \frac{\partial(\rho \nu \mu)}{\partial x}+\frac{\partial\left(\rho v^{2}\right)}{\partial y}+\frac{\partial(\rho \mu \omega)}{\partial z}=-\frac{\partial p}{\partial y}+\frac{\partial \sigma_{y x}}{\partial x}+\frac{\partial \sigma_{y y}}{\partial y}+\frac{\partial \sigma_{y z}}{\partial z} \\
& \frac{\partial(\rho \omega \mu)}{\partial x}+\frac{\partial(\rho \omega v)}{\partial y}+\frac{\partial\left(\rho \omega^{2}\right)}{\partial z}=-\frac{\partial p}{\partial z}+\frac{\partial \sigma_{z x}}{\partial x}+\frac{\partial \sigma_{z y}}{\partial y}+\frac{\partial \sigma_{z z}}{\partial z}
\end{aligned}
$$

where $p$ is the pressure of micro volume of fluid.

The energy equation:

$$
\begin{aligned}
& \frac{\partial(\rho \mu h)}{\partial x}+\frac{\partial(\rho v h)}{\partial y}+\frac{\partial(\rho \omega h)}{\partial z}= \\
& \frac{\partial}{\partial x}\left(k \frac{\partial T}{\partial x}\right)+\frac{\partial}{\partial y}\left(k \frac{\partial T}{\partial y}\right)+\frac{\partial}{\partial z}\left(k \frac{\partial T}{\partial z}\right)+u \frac{\partial P}{\partial x}+v \frac{\partial P}{\partial y}+w \frac{\partial P}{\partial z}+\Phi
\end{aligned}
$$

where $\mathrm{h}$ is specific enthalphy, $\mathrm{k}$ is thermal conductivity, $\Phi$ is dissipative terms.

$$
\begin{aligned}
\Phi=\sigma_{x x} \frac{\partial u}{\partial x} & +\sigma_{x y} \frac{\partial u}{\partial y}+\sigma_{x z} \frac{\partial u}{\partial z}+\sigma_{y x} \frac{\partial v}{\partial x}+\sigma_{y y} \frac{\partial v}{\partial y} \\
& +\sigma_{x z} \frac{\partial v}{\partial z}+\sigma_{z x} \frac{\partial w}{\partial x}+\sigma_{z y} \frac{\partial w}{\partial y}+\sigma_{z z} \frac{\partial w}{\partial z}
\end{aligned}
$$

The differential equations composed of formula (1), (2) and (3) are closed. The thevelocity field and temperature field can be obtained by means of numerical solution method.

\subsection{Mathematical Model of Fluid-Solid Coupling}

When the hydraulic retarder works, the transient flow field in the working cavity applies unsteady fluid force in blades, so that the blades produce deformation and vibration. The deformation and vibration effect on flow field in turn, so that the flow state of flow field changes. This interaction will produce a wide variety of fluid-solid coupling phenomenons. Based on Newton's laws of motion, using the finite element method, the dynamic equations of structure can be set up.

According to the coupling mechanism, the problems about fluid-solid coupling can be divided into two categories [8]. The first class of problems are that the coupling effect occurs only at the boundary between fluid and solid. The second class of problems are that the fluid domain and solid domain part or all overlap together, which are difficult to separate. According to the working characteristics of hydraulic retarder, the fluid in working chamber and the blades interact each other in the interface of fluid-solid coupling. The flow field applies a transient load on the blades, the deformation of blades in turn affect the distribution of flow field, then the output characteristics of hydraulic retarder are changed. Therefore, the fluid-solid coupling of hydraulic retarder belongs to the first class problems, whose coupling effect can be achieved by differential equations.

$$
M_{S} \ddot{r}+C_{s} \dot{r}+K_{s} r+f_{p}+f_{0}=0
$$

where $r$ is the displacement vector, $\mathrm{C}_{\mathrm{s}}$ is the damping matrix, $M_{s}$ is the mass matrix, $K_{s}$ is the stiffness matrix, $f_{p}$ is the node vector of fluid power in the interface between fluid and solid, $\mathrm{f}_{0}$ are other external excitation vectors in addition to $\mathrm{f}_{\mathrm{p}}$.

In the interface between fluid and solid, the pressure distribution of fluid element in the Formula (2) can be dispersed approximately as follows.

$$
p^{*(e)}=N_{e}^{T} p_{e}
$$

where $p_{e}$ is the node pressure vector of fluid element, $\mathrm{N}_{\mathrm{e}}$ is the shape function vector of fluid element.

Because the finite element equations of fluid-solid coupling of hydraulic retarder belong to the nonlinear partial differential equations, the methods used to solve the iterative solution of linear algebraic equations are adopted. Using the integrated solution method, the motion equations of structure and fluid are combined with each other. Thus, the unified equations of fluid-solid coupling system are formed, and the unified numerical methods are adopted to solve simultaneously. Therefore, the integral differential equations formed by fluid equations and structural equations are shown as follows.

$$
F(x)=\left\{\begin{array}{c}
F_{f}\left[x_{f}, d_{s}\left(x_{s}\right)\right] \\
F_{s}\left[x_{s}, \tau_{f}\left(x_{f}\right)\right]
\end{array}\right\}
$$

where $\mathrm{x}=\left(\mathrm{x}_{\mathrm{f}}, \mathrm{x}_{\mathrm{s}}\right), \mathrm{x}_{\mathrm{f}}$ is the vector waiting for solving of fluid equations, $x_{s}$ is the vector waiting for solving of structural equations, $F_{s}$ is the structural equations, and $F_{f}$ is the fluid equations.

The variables of fluid equations include the displacement variable $d_{s}$ of structure, and the variables of structural equations include the stress variable $\tau_{\mathrm{f}}$ of fluid. It can be seen that the fluid equations and the structure equations are coupled.

Using the Newton-Raphson method, the formula (7) is solved. The result is shown as follows.

$x_{k+1}=x_{k}-\left[\frac{\partial F(x)_{k}}{\partial x}\right]^{-1} F(x)_{k}$

The partial derivative of formula (8):

$$
\frac{\partial F(x)}{\partial x}=\left[\begin{array}{c}
\frac{\partial F(x)_{f}}{\partial x_{f}} \frac{\partial F(x)_{f}}{\partial x_{s}} \\
\frac{\partial F(x)_{s}}{\partial x_{f}} \frac{\partial F(x)_{s}}{\partial x_{s}}
\end{array}\right]=\left[\begin{array}{c}
T_{f f} T_{f s} \\
T_{s f} T_{s s}
\end{array}\right]
$$


$\mathrm{x}_{\mathrm{k}+1}$ expresses the next iteration value.

$$
x_{k+1}=x_{k}+\Delta x_{k}=\left[\begin{array}{c}
\Delta x_{f, k} \\
\Delta x_{s, k}
\end{array}\right]
$$

where $\Delta \mathrm{x}_{\mathrm{f} . \mathrm{k}}$ is the variable in fluid equations, $\Delta \mathrm{x}_{\mathrm{s}, \mathrm{k}}$ is the variable in structure equations.

It can be obtained as follows by the formula $(8) \sim(10)$.

$$
\left[\begin{array}{c}
F_{f, k} \\
F_{s, k}
\end{array}\right]=-\left[\begin{array}{c}
T_{f f} T_{f s} \\
T_{s f} T_{s s}
\end{array}\right]\left[\begin{array}{c}
\Delta x_{f, k} \\
\Delta x_{s, k}
\end{array}\right]
$$

In order to improve the constringency of numerical solutions of fluid-solid coupling in the formula (11), the relaxation coefficient $\lambda$ is added to the formula (9), and substituted into the formula (11). It can be got as follows.

$$
\left[\begin{array}{c}
F_{f, k} \\
F_{s, k}
\end{array}\right]=-\left[\begin{array}{c}
T_{f f} \lambda_{d} T_{f s} \\
T_{s f} \lambda_{\tau} T_{s s}
\end{array}\right]\left[\begin{array}{c}
\Delta x_{f, k} \\
\Delta x_{s, k}
\end{array}\right]
$$

where $\lambda_{\mathrm{d}}$ is the relaxation coefficient of structure displacement, $\lambda_{\tau}$ is the relaxation coefficient of fluid stress.

\section{SIMULATION OF HEAT-FLUID COUPLING OF HYDRAULIC RETARDER}

In this paper, the effective diameter of trus section of hydraulic retarder is $465 \mathrm{~mm}$, the cavity shape of working chamber is oblate, the blades of driving wheel and fixed wheel are straight blades whose front rake is $50^{\circ}$, the spacing of driving wheel and fixed wheel is $4 \mathrm{~mm}$.

Based on CATIA software, the three dimensional model of hydraulic retarder is established. The whole flow passage model of hydraulic retarder is extracted on the basis of the three dimensional model established. The geometry model of liquid channels of working chamber is shown in Fig. (1).

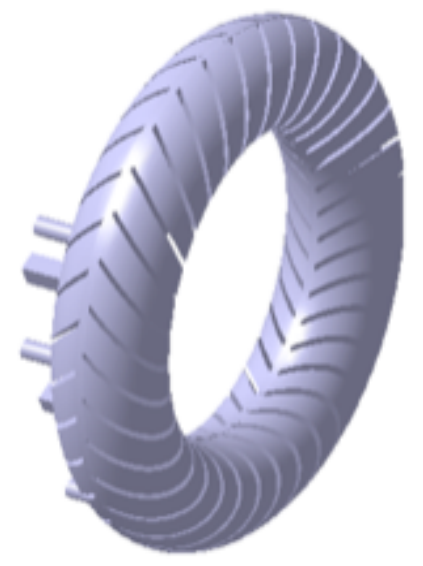

Fig. (1). Geometric model of flow passage extracted.

The mesh of whole flow passage model is divided by using ICEM software, and the total number of grid cells obtained is 652552 . The mesh model of whole flow passage is shown in Fig. (2).

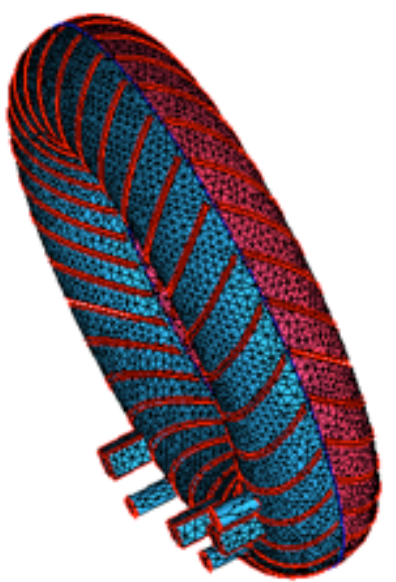

Fig. (2). Mesh model of whole flow passage.

\subsection{Setting of Boundary Conditions}

In the simulation of hydraulic retarder, according to the flow model and internal flow, the following three kinds of boundary conditions are set mainly. They are the boundary conditions of inlet and outlet, the boundary conditions of wall and the boundary conditions in the interface between driving wheel and fixed wheel.

The boundary conditions of inlet and outlet refer to the situation of appointing flow variables in the boundary of inlet and outlet. The inlets and outlets of hydraulic retarder are located in the outer race of fixed wheel. In the process of CFD numerical simulation, the boundary conditions of inlet are set to velocity-inlet, and the boundary conditions of outlet are set to outflow.

The outer ring surfaces of driving wheel and fixed wheel and the blade's surfaces are not deformable walls in the calculation area. Because the fixed wheel is fixed and the driving wheel rotates with a certain angular velocity, the outer ring surface of driving wheel and the blade's surfaces of driving wheel are set up to the moving walls whose speed of revolution is $0 \mathrm{r} / \mathrm{min}$ relative to the calculation region of driving wheel, and the outer ring surface of fixed wheel and the blade's surfaces of fixed wheel are set up to the stationary walls.

Because of the interaction between driving wheel and fixed wheel of hydraulic retarder, the working fluid flows in and out in the interface at the same time, and the internal flow is very complex. In this paper, in order to take the fixed wheel and the driving wheel into a computational domain for simulation analysis. Using the sliding mesh method, the flow in the interface between fixed wheel and driving wheel is dealed with. The specific boundary conditions are shown as Fig. (3).

\subsection{Simulation Analysis}

Because the distribution of temperature field and flow field under different speed are similar when the hydraulic retarder is filled with fluid. In this paper, the temperature distribution and flow field distribution in the working cavity of hydraulic retarder and the surface of blade are mainly 
analysed to hydraulic retarder in the state of whole filling when the rotate speed is $1600 \mathrm{r} / \mathrm{min}$.

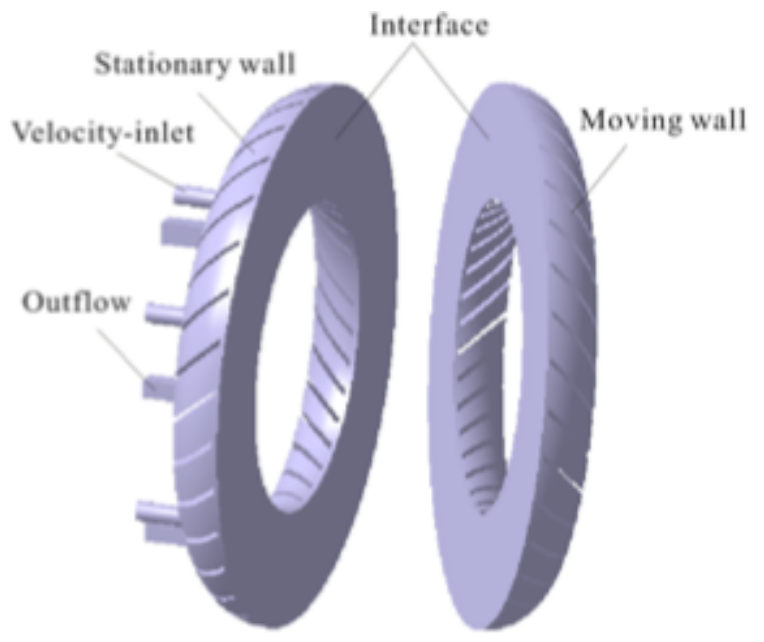

Fig. (3). Setting of boundary conditions.

The full passage way is selected as the computational region, the sliding mesh technique is used to solve the mutual interaction between fixed wheel and driving wheel during the calculation of fluid. The turbulence model applies the standard $\mathrm{k}-\varepsilon$ model, the coupling algorithm of velocity and pressure uses the SIMPLEC algorithm, the spatial discretization adopts the discrete differential equations of two order scheme, the iterative calculation of algebraic equation takes secondary relaxation. After initializing the flow field in the solver and setting the step size of time and the number of iterations at the same time, the iterative calculation is carried out.

When the residual value is satisfied with the convergence criterion, then the calculation is accomplished. Otherwise, the iterative calculation continues until it reaches the convergence criterion. Finally, the internal flow field and temperature field of hydraulic retarder are obtained according to the output of calculation results. The temperature field distribution of hydraulic retarder is shown as Fig. (4).

It can be seen from Fig. (4) that the highest temperature of hydraulic retarder is $391 \mathrm{~K}$ when the hydraulic retarder is filled with fluid. The temperature of outer ring of fixed wheel is higher than the temperature of its inner ring, but the temperature of outer ring of driving wheel is lower than the temperature of its inner ring. This is because the liquid of working chamber suffers the effect of blades of driving wheel and the centrifugal force, the mechanical energy of driving wheel is transformed into the kinetic energy of working fluid. The liquid of working cavity is incompressible viscous liquid. Therefore, the velocity gradient of liquid is greater, the internal friction of moleculars is greater, and the heat generated is more. In the inner ring of driving wheel, the velocity of working liquid is low and there is a big difference with the speed of driving wheel, which leads to higher temperature in the inner ring of driving wheel. When the working liquid gradually moves from the inner ring to the outer ring of driving wheel, the velocity of working liquid raises with the increase of the radius, so the heat generated is on the decrease. Similarly, when the working liquid with very high energy rushes from the exits of driving wheel to the static fixed wheel, the more heat is produced because of the speed difference between the working liquid and the fixed wheel. When the working liquid flows back to the entrances of fixed wheel, the heat become smaller.
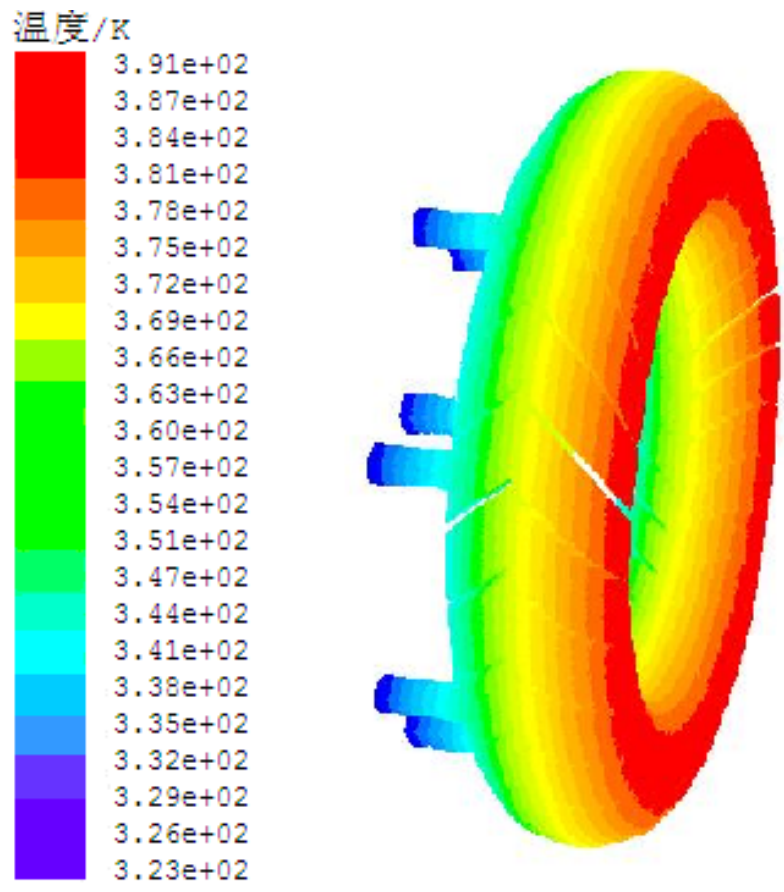

Fig. (4). Temperature field distribution of hydraulic retarder.

When the hydraulic retarder works, the working liquid circulates in the flow passage. The fluid of hydraulic retarder forms two special surfaces in a flow cell, which are the pressure surface and the suction surface. The pressure surface is the surface effected directly by working liquid, and is main working surface when the hydraulic retarder works. The suction surface is the surface which is not effected directly by working liquid. The static pressure distribution nephograms of suction surface and pressure surface of driving wheel are shown as Figs. $(\mathbf{5}, \mathbf{6})$ respectively.
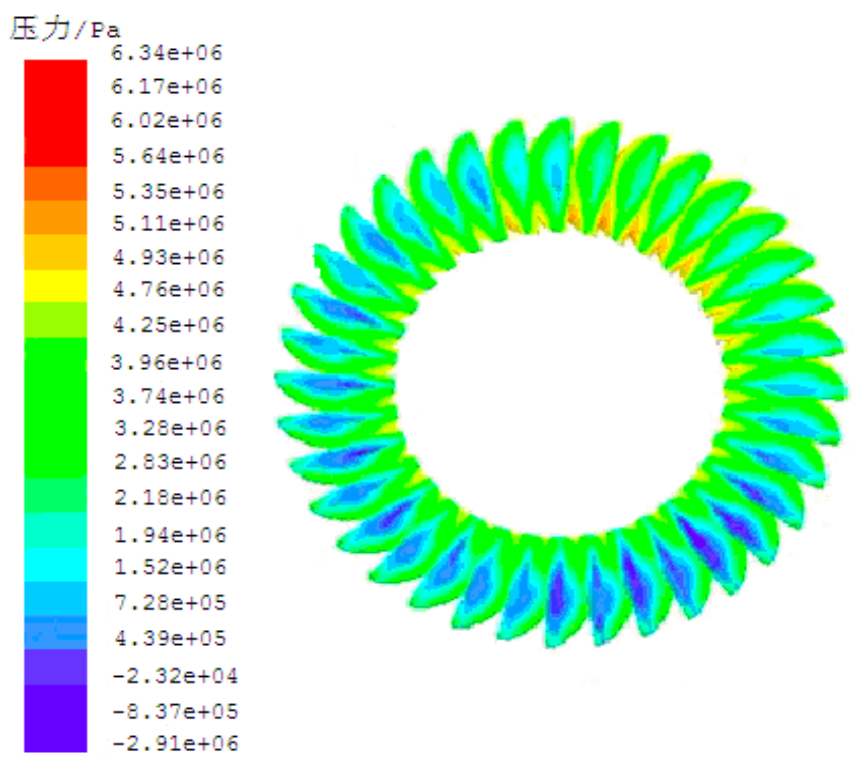

Fig. (5). Distribution map of suction surface of driving wheel. 
It can be seen that the pressure distribution of suction surface of driving wheel displays as a decreasing trend from the outer ring of blade to the center of circle from Fig. (5). However, there is a large area of low pressure at the leading edge of blades. This pressure gradient is mainly generated by the centrifugal force of working liquid. The zone of continuous negative pressure appears in the leading edge of some blades, which is mainly because this region corresponds to the oil inlet position of fixed wheel. The fluid with high speed rushes into working chamber, the low pressure zone is produced within this area, and it leads to the generation of part hydraulic loss.

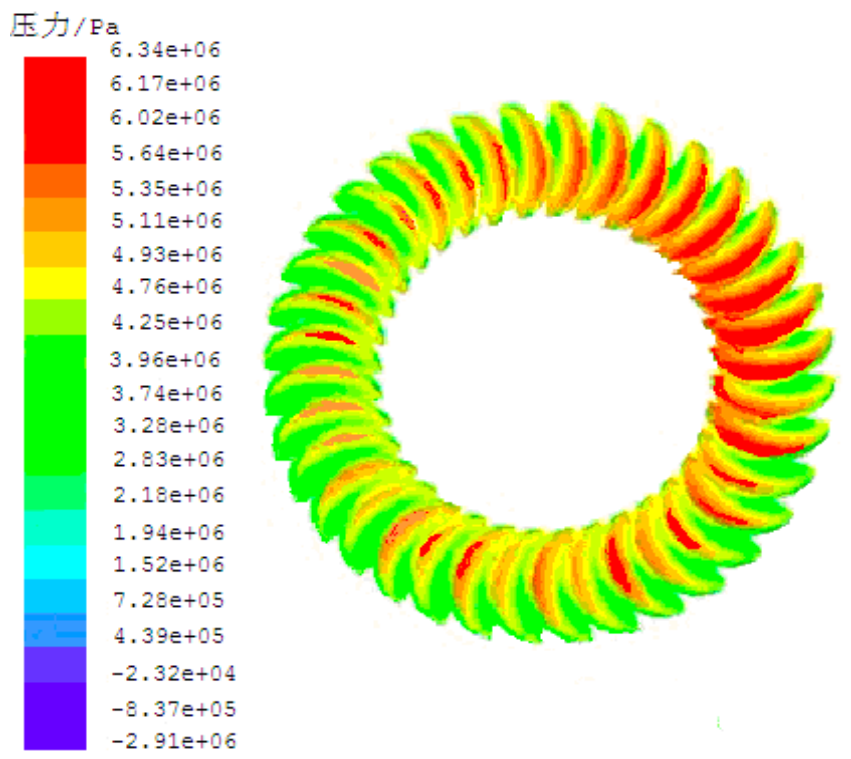

Fig. (6). Distribution map of pressure surface of driving wheel.

It can be seen that the region of high pressure of pressure surface is more than suction surface, because the pressure surface of driving wheel is taken as the main working plane from Fig. (6). During working process, because the pressure surface of driving wheel suffers the enormous impact of working liquid, the velocity of liquid decreases rapidly and a large range of high pressure area is formed on the pressure surface. Due to the larger impact, the entrance of working liquid also produces a local high pressure zone. At the same time, the outer ring also produces high pressure under the action of centrifugal force. The static pressure distribution of each different channel is roughly same, just the change scope of respective pressure is different.

The static pressure distribution nephograms of suction surface and pressure surface of fixed wheel are shown as Fig. (7) and Fig. (8) respectively. It can be seen that the static pressure distribution trends of suction surface and pressure surface of fixed wheel are similar to the driving wheel from Figs. $(\mathbf{7}, \mathbf{8})$. The static pressure distribution of suction surface of fixed wheel displays as a decreasing trend from the outer ring of blade to the center of circle, and there is a large area of low pressure at the leading edge of blades. Because the three oil inlets of hydraulic retarder are arranged in the vicinity of the leading edge of fixed wheel's blades, and the blades near oil inlets are relatively thick. At the same time, because the liquid rushes into working chamber with high speed from the inlets of hydraulic retarder, the larger area of negative pressure appears in the leading edge of part blades.

It can be seen that the pressure distribution of pressure surface of fixed wheel is similar to the driving wheel from Fig. (8). The pressure gradually diminishes from the outer ring of blade to the center of circle, and the value of pressure in the center of circle is minimum. The speed of working fluid increases after absorbing energy from the inside of driving wheel. The fluid with high speed directly impacts the pressure surface of fixed wheel. Due to the hindrance of fixed wheel, a large number of shock losses are produced and a wide range of high pressure area is formed. In the working process of hydraulic retarder, the mechanical energy of driving wheel is transformed into the kinetic energy of liquid. The fluid with high energy impacts to the pressure surface of fixed wheel, and a large area of high pressure is formed. The part of high pressure zone is produced near the outer ring at the root of blades, because the fluid which flows from the driving wheel has higher kinetic energy.

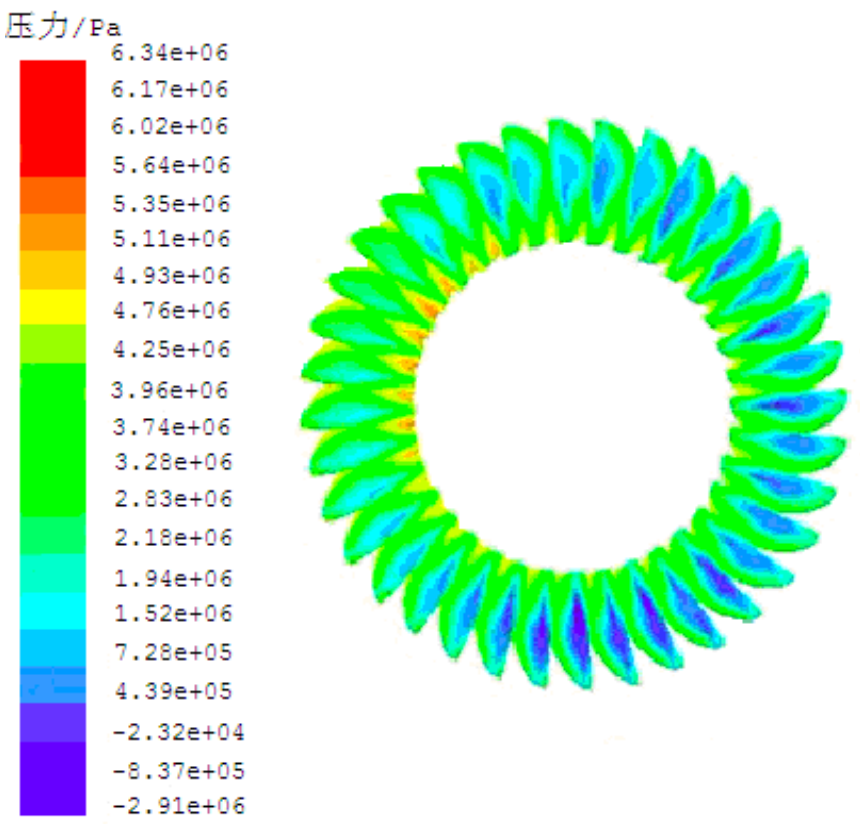

Fig. (7). Distribution map of suction surface of fixed wheel.

\subsection{Simulation of Fluid-Solid Coupling of Hydraulic Retarder}

The temperature field and pressure field of fluid obtained by the analysis in FLUENT software are taken as the boundary conditions, which are applied to the blades of fixed wheel and driving wheel. And the centrifugal forces are separately applied on the fixed wheel and the driving wheel, then the analysis of fluid-solid coupling is carried out to hydraulic retarder. When the hydraulic retarder is filled with liquid, the deformation contour map of fixed wheel under the coupling action of centrifugal force and working fluid together is shown as Fig. (9). It can be seen from Fig. (9) that the deformation of fixed wheel gradually becomes larger with the increase of rotating radius. The maximum deformation of fixed wheel appears in the outer end face of fixed wheel and its value is about $0.011455 \mathrm{~mm}$ when the hydraulic retarder work. The deformation of blade of fixed 
wheel becomes smaller gradually along the direction of cavity.

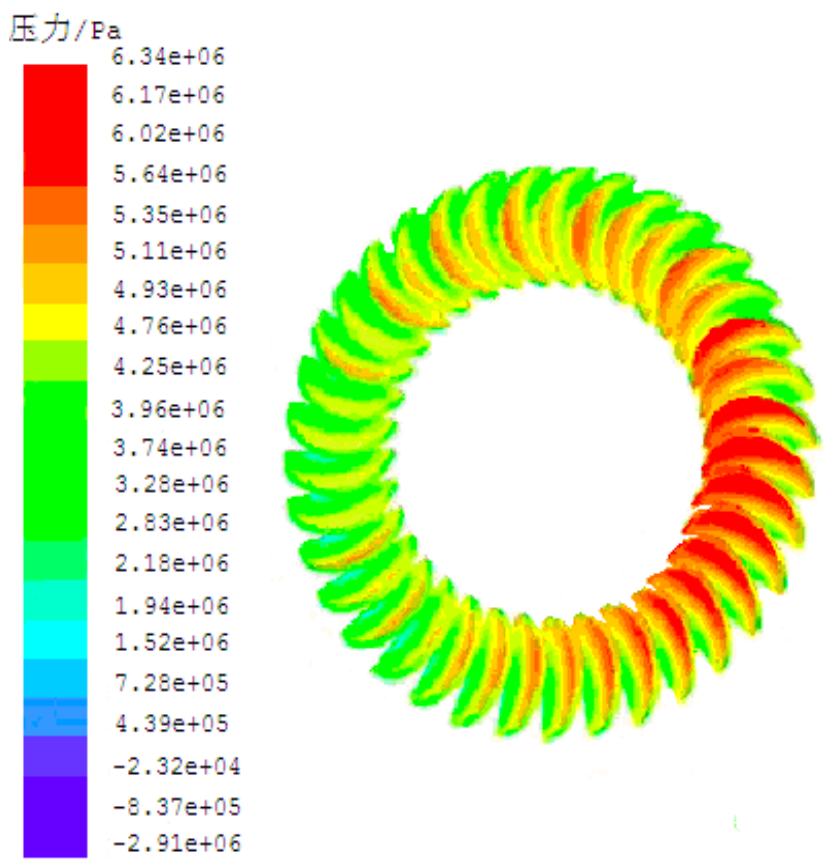

Fig. (8). Distribution map of pressure surface of fixed wheel.

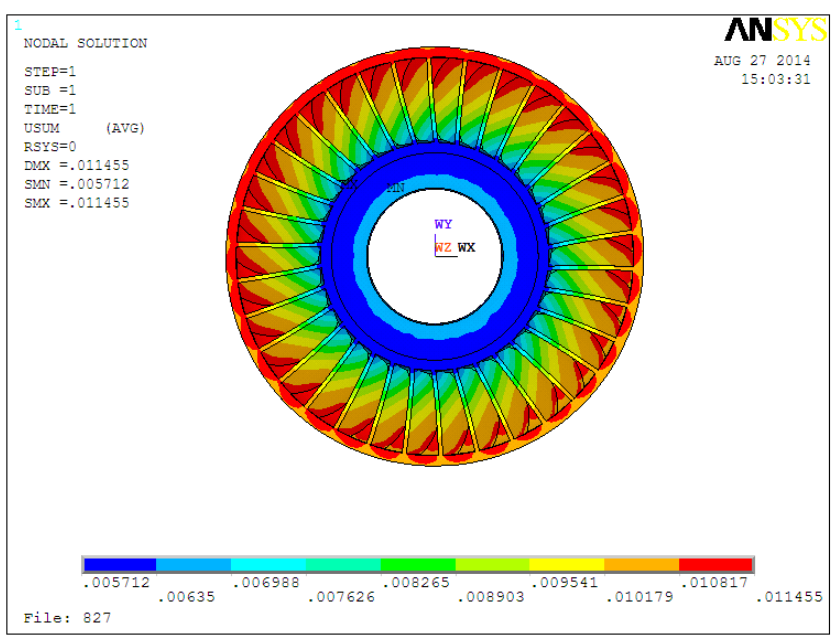

Fig. (9). Deformation contour map of fixed wheel.

The stress contour map of fixed wheel under the coupling action of centrifugal force and working fluid together is shown as Fig. (10). The areas of larger stress focus on the blades, and present ring distribution of the equivalent stress. The stresses on the blades are uniform. Because the fixed wheel undergoes the interaction of centrifugal force, the rigid constraint position of shaft hole of fixed wheel is under tensile stress, and appears the phenomenon of stress concentration. There is stress concentration at the root of blades corresponding to the inlets and outlets of fixed wheel. In the process of stirring fluid, the working plane of fixed wheel's blade is under pressure, and the non working plane of fixed wheel's blade is under tensile stress. The stress distribution on the blade becomes smaller gradually from the leaf end along the axial direction.

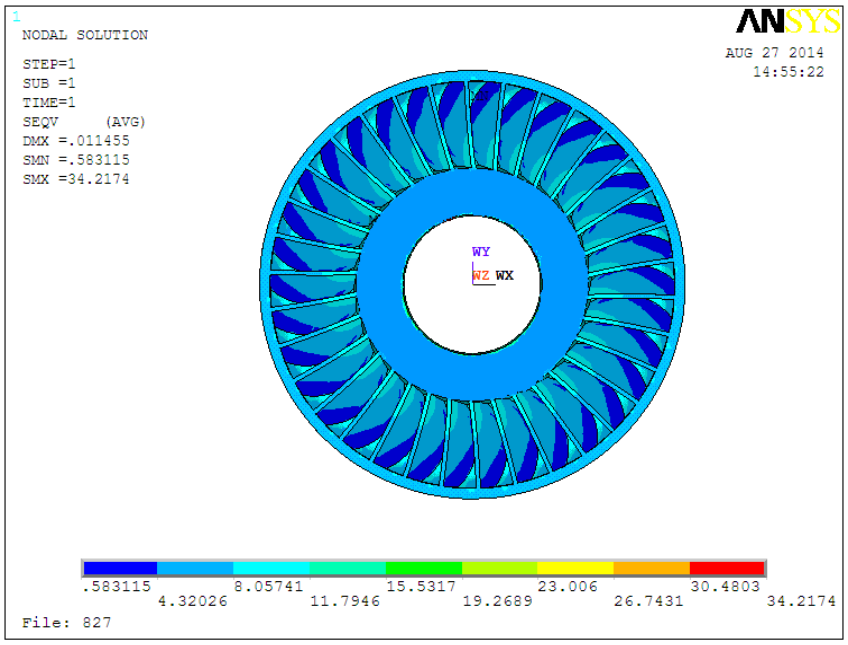

Fig. (10). Stress contour map of fixed wheel.

When the hydraulic retarder is filled with liquid, the deformation contour map of driving wheel under the coupling action of centrifugal force and working fluid together is shown as Fig. (11). It can be seen from Fig. (11) that the deformation of driving wheel gradually becomes larger with the increase of rotating radius. But, the maximum deformation of driving wheel appears on the blade of driving wheel and its value is about $0.019624 \mathrm{~mm}$, which is higher than the deformation of fixed wheel's blade. This is because the pressure, which the working fluid impacts the blade of driving wheel, plays a main role on the deformation of driving wheel's blade. The maximum deformation of driving wheel' blade appears on the center area of external end face of blade.

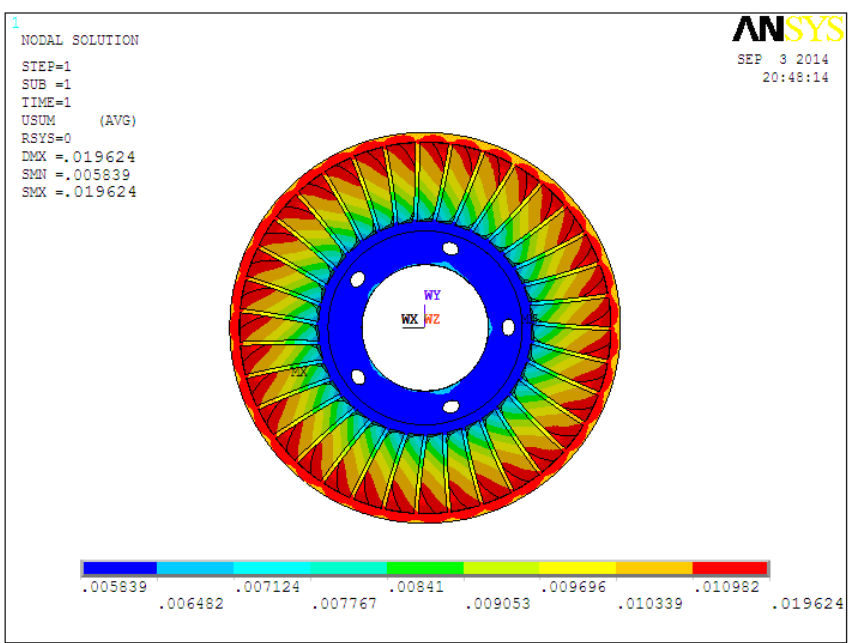

Fig. (11). Deformation contour map of driving wheel.

The stress contour map of driving wheel under the coupling action of centrifugal force and working fluid together is shown as Fig. (12). The maximum stress effects on the driving wheel's blade, and at the same time, there is larger stress in the rigid constraint position of shaft hole of driving wheel. The phenomenon of stress concentration appears at the root of blades corresponding to the inlets and outlets of driving wheel. Meanwhile, there is greater stress in the blade near the region. The fluid with high speed which 
flows from the entrances of fixed wheel impacts the blades of driving wheel. In the process of stirring fluid, the working plane of driving wheel's blade is under pressure, and the non working plane of driving wheel's blade is under tensile stress. The stress of working surface of blade is large in the outer end, and is small in the center region. While the stress distribution of non working surface is more uniform.

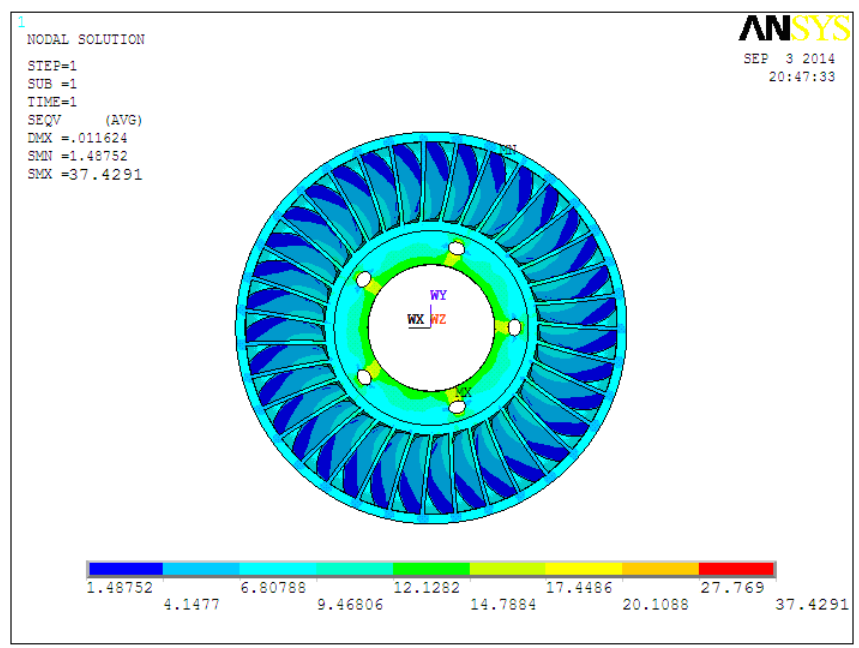

Fig. (12). Stress contour map of driving wheel.

\section{CONCLUSION}

In this paper, the three dimensional geometric model of hydraulic retarder is established based on CATIA software, and the whole flow passage model of hydraulic retarder is extracted on the basis of the three dimensional model established. The CFD calculation and finite element numerical simulation are carried out to hydraulic retarder by using the multi-field coupling method of heat, fluid and solid, the distributions of temperature field, pressure field and stress field of hydraulic retarder are obtained, and the performance of hydraulic retarder is forecasted. The results of analysis show that the speed has great influence to the stress distribution of blade and the effect of fluid pressure on blade is very significant when the hydraulic retarder is filled with fluid. Therefore, it is needed to ensure the strength of blade's root in the process of design to avoid stress concentration. These provide the theoretical foundation and references for optimization design and performance prediction of hydraulic retarder.

\section{CONFLICT OF INTEREST}

The authors confirm that this article content has no conflict of interest.

\section{ACKNOWLEDGEMENTS}

This research is supported by the University Science Research Project of Jiangsu Province (13KJB580005), the Jiangsu Province Ordinary University Graduate Research and Innovation Project (CXZZ13_0659), and the Open Fund Project of Automotive Engineering Key Laboratory of Jiangsu Province (QC201207).

\section{REFERENCES}

[1] G. Zhao, and M. Wei, "Quantitative evaluation on the braking stability of the composite braking system composed of a retarder and a service-brake system", Acta Armamentarii, vol. 2, pp. 185189, February 2009.

[2] D. Wang, "Application and developing prospect of the heavy duty truck retarder", Autom. Technol., vol. 6, pp. 17-19, June 2012.

[3] C. Boivin, and C. Ollivier, "A toolkit for numerical simulation of PDEs: II. Solving generic multiphysics problems", Comput. Method Appl. M, vol. 36, pp. 3891-3918, December 2004.

[4] C. Boivin, and C. Ollivier, "A toolkit for numerical simulation of PDEs: I. Fundamentals of generic finite-volume simulation", Comput. Method. Appl. M, vol. 9, pp. 1147-1175, March 2003.

[5] L. Fan, W. Ma, and W. Cai, "Thermal-hydraulic analysis of hydrodynamic coupling”, AISS Adv. Inform. Sci. Serv. Sci., vol. 4, pp. 393-399, April 2012.

[6] E. Mostafa, I. B. Lee, and S. H. Song, "Computational fluid dynamics simulation of air temperature distribution inside broiler building fitted with duct ventilation system", Biosystems Engineering, vol. 4, pp. 293-303, April 2012.

[7] Z. Khatir, J. Paton, and H. Thompson, "Computational fluid dynamics (CFD) investigation of air flow and temperature distribution in a small scale bread-baking oven", Appl. Energy, vol. 1, pp. 89-96, January 2012.

[8] J. Xing, S. Zhou, and E. Cui, "A survey on the fluid-solid in teraction mechanics", Adv. Mech, vol. 1, pp. 19-38, January 1997. 